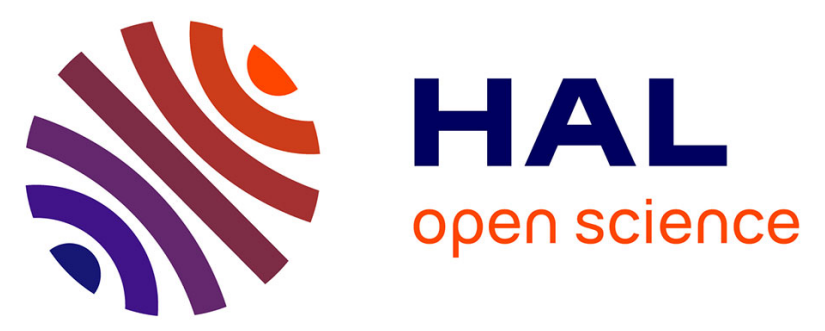

\title{
Determination of spin Hall angle, spin mixing conductance, and spin diffusion length in CoFeB/Ir for spin-orbitronic devices
}

\author{
T. Fache, J Rojas-Sánchez, L. Badie, S Mangin, S. Petit-Watelot
}

\section{- To cite this version:}

T. Fache, J Rojas-Sánchez, L. Badie, S Mangin, S. Petit-Watelot. Determination of spin Hall angle, spin mixing conductance, and spin diffusion length in $\mathrm{CoFeB} / \mathrm{Ir}$ for spin-orbitronic devices. Physical Review B, 2020, 102, 10.1103/physrevb.102.064425 . hal-02999938

\section{HAL Id: hal-02999938 \\ https://hal.univ-lorraine.fr/hal-02999938}

Submitted on 11 Nov 2020

HAL is a multi-disciplinary open access archive for the deposit and dissemination of scientific research documents, whether they are published or not. The documents may come from teaching and research institutions in France or abroad, or from public or private research centers.
L'archive ouverte pluridisciplinaire HAL, est destinée au dépôt et à la diffusion de documents scientifiques de niveau recherche, publiés ou non, émanant des établissements d'enseignement et de recherche français ou étrangers, des laboratoires publics ou privés. 


\title{
Determination of spin Hall angle, spin mixing conductance, and spin diffusion length in $\mathrm{CoFeB/Ir}$ for spin-orbitronic devices
}

\author{
T. Fache $\odot$, J. C. Rojas-Sanchez $\odot{ }^{*}$ L. Badie, S. Mangin, and S. Petit-Watelot ${ }^{\dagger}$ \\ Université de Lorraine, CNRS, Institut Jean Lamour, F-54000 Nancy, France
}

(Received 6 May 2020; revised 5 August 2020; accepted 10 August 2020; published 25 August 2020)

\begin{abstract}
Iridium is a very promising material for spintronic applications due to its interesting magnetic properties such as large Ruderman-Kittel-Kasuya-Yosida exchange coupling as well as its large spin-orbit coupling value. Ir is for instance used as a spacer layer for perpendicular synthetic antiferromagnetic or ferrimagnet systems. However, only a few studies of the spintronic parameters of this material have been reported. In this paper, we present inverse spin Hall effect-spin pumping ferromagnetic resonance measurements on $\mathrm{CoFeB} / \mathrm{Ir}$ based bilayers to estimate the values of the effective spin Hall angle, the spin diffusion length within iridium, and the spin mixing conductance in the $\mathrm{CoFeB} / \mathrm{Ir}$ bilayer. In order to have reliable results, we performed the same experiments on $\mathrm{CoFeB} / \mathrm{Pt}$ bilayers, whose behavior is well known due to numerous reported studies. Our experimental results show that the spin diffusion length within iridium is $1.3 \mathrm{~nm}$ for resistivity of $250 \mathrm{n} \Omega \mathrm{m}$, the spin mixing conductance $g_{\text {eff }}^{\uparrow \downarrow}$ of the $\mathrm{CoFeB} / \mathrm{Ir}$ interface is $30 \mathrm{~nm}^{-2}$, and the spin Hall angle of iridium has the same sign as the one of platinum and is evaluated at $26 \%$ of the one of platinum. The value of the spin Hall angle found is $7.7 \%$ for Pt and $2 \%$ for Ir. These relevant parameters shall be useful to consider Ir in new concepts and devices combining spin-orbit torque and spin-transfer torque.
\end{abstract}

DOI: 10.1103/PhysRevB.102.064425

\section{INTRODUCTION}

Iridium is a very promising material for spintronic applications. Its properties include large spin-orbit coupling [1], large Ruderman-Kittel-Kasuya-Yosida (RKKY) exchange coupling [2], and strong interface contribution to perpendicular magnetic anisotropy (PMA) [3-6] and to interfacial Dzyaloshinskii-Moriya interaction [7]. Ir has been shown to be a key element as a spacer layer to create model perpendicular synthetic ferrimagnets or synthetic antiferromagnets $[8,9]$. However, to our knowledge, the spin Hall effect, which has proven to be an efficient physical effect to manipulate magnetization [10-16], was scarcely studied in Ir $[7,17,18]$. Given the scattering among the spin transport measurements, and spin-Hall effect (SHE) characterizations, we have decided to perform a comparative study. Indeed, in this paper, we display the results of spin pumping voltage induced ferromagnetic resonance (SP-FMR) experiments obtained on $\mathrm{Co}_{40} \mathrm{Fe}_{40} \mathrm{~B} / \mathrm{Ir}$ and $\mathrm{Co}_{40} \mathrm{Fe}_{40} \mathrm{~B} / \mathrm{Pt}$ based bilayers, as it has been proven that SP-FMR is one of the most effective experiments in order to probe the inverse spin-Hall effects (ISHE) [19-21]. We characterized the SHE for iridium layers in comparison to $\mathrm{Pt}$, whose spin Hall behavior is already well known [22-35]. Therefore, we propose here a comparative approach to determine the spin-to-charge current conversion efficiency. We present our experimental determination of the spin diffusion length $\left(l_{s f}\right)$, the effective spin mixing conductance $\left(g_{\text {eff }}^{\uparrow \downarrow}\right)$, and the effective spin Hall angle $\left(\theta_{S H E}\right)$ for iridium based materials.

\footnotetext{
${ }^{*}$ Corresponding author: juan-carlos.rojas-sanchez@univlorraine.fr

${ }^{\dagger}$ Corresponding author: sebastien.petit-watelot@univ-lorraine.fr
}

\section{Samples growth and SP-FMR measurements}

The samples used in our experiments are $\mathrm{Si}_{-} \mathrm{SiO}_{2}(300$ $\mathrm{nm}) / / \mathrm{CoFeB}(5 \mathrm{~nm}) / \mathrm{Ir}$ and $\mathrm{Si}_{-} \mathrm{SiO}_{2}(300 \mathrm{~nm}) / / \mathrm{CoFeB}(5 \mathrm{~nm}) / \mathrm{Pt}$ bilayers deposited on thermally oxidized silicon substrates. The double slash, //, stands for the position of the substrate. We chose to grow samples with various iridium or platinum capping thickness $(1,2,4,6$, and $15 \mathrm{~nm})$ but keeping the same 5-nm $\mathrm{Co}_{0.4} \mathrm{Fe}_{0.4} \mathrm{~B}_{0.2}$ ferromagnetic layer thickness. All the layers were deposited by magnetron sputtering with a base vacuum pressure of $8 \times 10^{-9} \mathrm{mbar}$, and an argon deposition pressure of $5 \times 10^{-3} \mathrm{mbar}$, from pure elemental targets for platinum and iridium, and from an alloy $\mathrm{Co}_{0.4} \mathrm{Fe}_{0.4} \mathrm{~B}_{0.2}$ target. The samples grown were then patterned into rectangular slabs with electrodes at the end to measure the spin pumping voltage. The devices were structured using standard optical lithography techniques. An antenna was patterned on top of the sample, which was insulated by a $\mathrm{SiO}_{2}$ cap of $75 \mathrm{~nm}$. In this antenna we injected a $\mathrm{GHz}$ rf current which generates the radio-frequency field $h_{\mathrm{rf}}$ on the sample. A static magnetic field ranging from 0 to $0.5 \mathrm{~T}$ can also be applied in plane. Depending on the value of the applied field, $h_{\mathrm{rf}}$ could excite the magnetization at resonance. Through the spin-pumping effect, the precession of the magnetization yield to the creation of a pure transverse spin current $j_{\mathrm{s}}$ that is injected from the $\mathrm{CoFeB}$ layer into the Ir or Pt layer. $j_{\mathrm{s}}$ is generally expressed as follows [19]:

$$
j_{S}=\frac{\hbar \operatorname{Re}\left(g^{\uparrow \downarrow}\right)}{4 \pi} \int_{0}^{\frac{2 \pi}{\omega}}\left(\boldsymbol{m} \times \frac{d \boldsymbol{m}}{d t}\right) d t,
$$

where $g^{\uparrow \downarrow}$ is the spin mixing conductance, $\boldsymbol{m}$ is the reduced magnetization, and $\omega$ is the rf field pulsation. This spin current injected in the heavy metal (HM) layer is then converted 


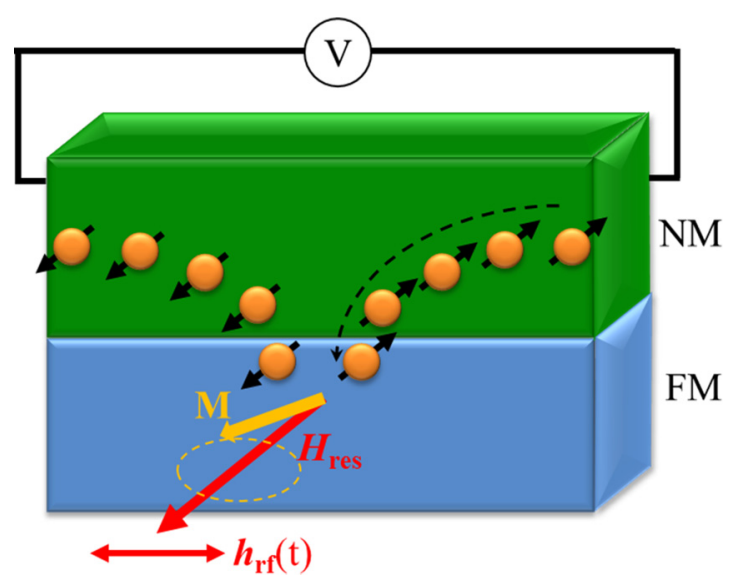

FIG. 1. Schematic of the bilayer sample composed of a ferromagnetic layer of $\mathrm{CoFeB}(\mathrm{FM})$ and a nonmagnetic material NM. The static field $H_{\text {res }}$ is applied perpendicularly to the wire, whereas the rf field $h_{\mathrm{rf}}$ is aligned with the wire's length. The magnetization $M$ precesses around $H_{\text {res }}$, generating a spin current polarized along $H_{\text {res }}$, which is injected in the NM layer. This injected spin current in the NM layer is converted into a charge current through the ISHE. In an open circuit we can detect the voltage $V$ as depicted. The charge current production is nothing else than the voltage amplitude normalized by the total resistance of the sample.

into a charge current due to the ISHE which is detected by electrical means, by measuring the voltage in an open circuit, as represented in Fig. 1. Such a voltage due to spin pumping is symmetrical around the resonance field as shown in Fig. 2(a). From the peaks at resonance, several materials properties can be deduced. For instance, from this ISHE spin pumping voltage, the resonance magnetic field $H_{\text {res }}$ as a function of the frequency $f$ of the rf excitation applied to the sample gave us information about the ferromagnetic layer excited.

The relationship between these parameters is described by the Kittel law [36], as presented in Fig. 2(b):

$$
f=\frac{\gamma \mu_{0}}{2 \pi} \sqrt{H_{\text {res }}\left(H_{\text {res }}+M_{\text {eff }}\right)},
$$

where $\gamma$ is the gyromagnetic ratio, $\mu_{0}$ is the vacuum permeability, and $M_{\text {eff }}$ is the effective saturation magnetization of the FM layer. $M_{\text {eff }}$ that is extracted does not change significantly with the thickness of the nonmagnetic material (here Pt or Ir) that caps the ferromagnetic layer. For all the samples grown, the value of $\mu_{0} M_{\mathrm{eff}}$ is evaluated at $1.39 \mathrm{~T}$.

On the other hand, from the measurement of the spin pumping voltage as a function of the applied field [Fig. 2(a)] one can determine the linewidth $\Delta H$ of the voltage peaks to estimate the magnetic damping $\alpha$ of the materials. The evolution of the linewidth as a function of the frequency [Fig. 2(c)] of the excitation shows a linear dependence, whose slope is found to be proportional to the effective damping, following the relationship [37]

$$
\mu_{0} \Delta H=\mu_{0} \Delta H_{0}+\frac{2 \pi \alpha}{\gamma} f,
$$

where $\Delta H$ is the linewidth of the ferromagnetic resonance peak and $\Delta H_{0}$ is the inhomogeneous broadening. An example of such damping determination is shown in Fig. 2(c). We
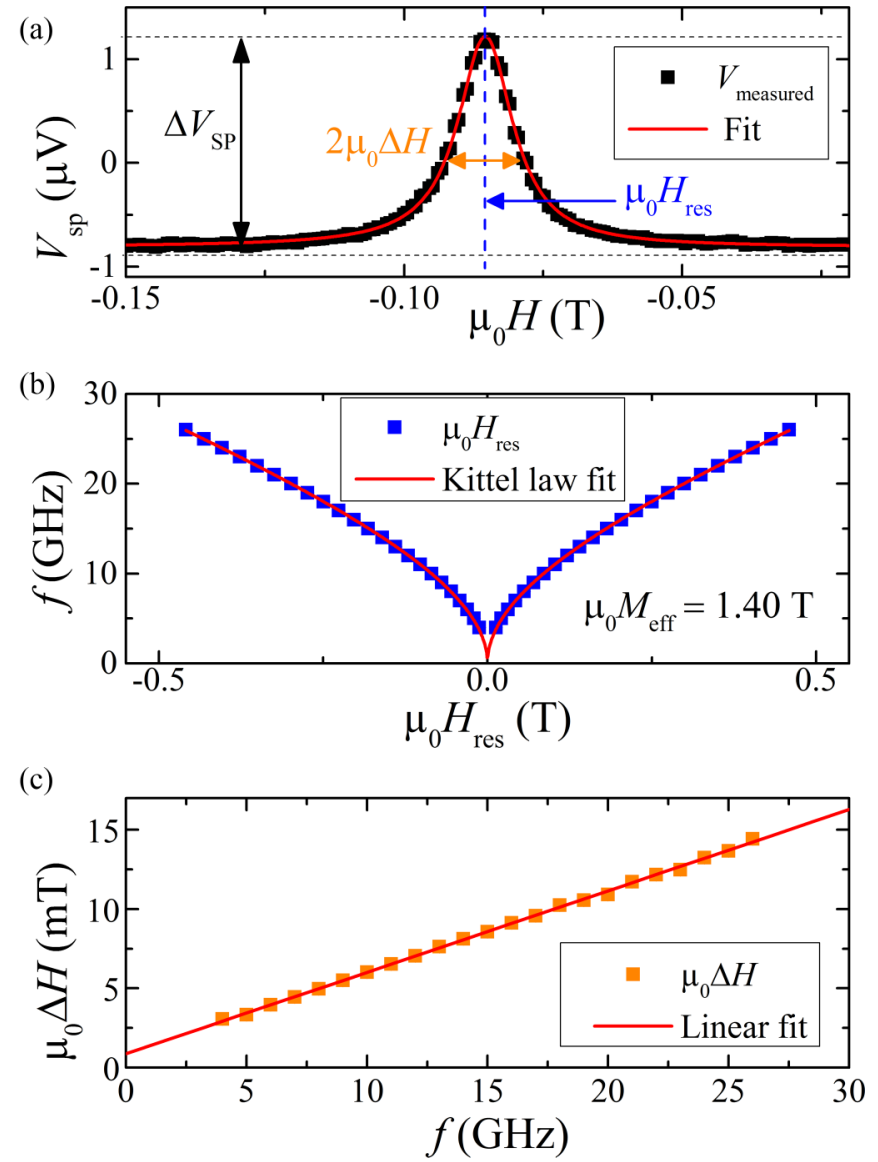

FIG. 2. Measurements on $/ / \operatorname{CoFeB}(5 \mathrm{~nm}) / \operatorname{Ir}(4 \mathrm{~nm})$. (a) Spin pumping voltage at a frequency of $10 \mathrm{GHz}$; (b) linewidth $\left(\mu_{0} \Delta H\right)$ as a function of the frequency; (c) frequency $\left(f_{\mathrm{r}}\right)$ vs the applied magnetic field $\left(\mu_{0} H\right)$. On all these graphs, symbols represent experimental measurements whereas solid red lines correspond to fits. In (c) the Kittel law, given by Eq. (2), yields $\mu_{0} M_{\text {eff }}=1.40 \mathrm{~T}$.

have performed such a damping determination on both series of samples. Figure 3 shows the evolution of the effective damping $\alpha$ as a function of the thickness of nonmagnetic material.

To measure the value of the intrinsic damping we used a reference sample of $\mathrm{CoFeB}(5 \mathrm{~nm})$ capped with $\mathrm{Al}(3 \mathrm{~nm})$. The intrinsic damping value $\alpha_{0}=7.5 \times 10^{-3}$ for $t_{N M}=0$ was obtained by a vector network analyzer (VNA)-FMR, since SP-FMR could not be an efficient detection method in that special case. Moreover, for $t_{N M}>0$ we have checked that the damping values obtained using both methods were consistent. The value of the intrinsic damping was found to be slightly higher than the values given in part of the literature $\left(4-5 \times 10^{-3}\right)[38-41]$. This could be explained by the growth conditions. Indeed, the studied carried out by Xu et al. [42] shows that the magnetic damping of sputtered $\mathrm{CoFeB}$ is very sensitive and decreases when the argon pressure increases. They reported a damping value of $13 \times 10^{-3}$ when the Ar pressure was 3 mTorr. The annealing also affects the damping $[38,41]$. Another possibility might be to attribute this slight difference to the aluminium oxide layer that caps the $\mathrm{CoFeB}$ 


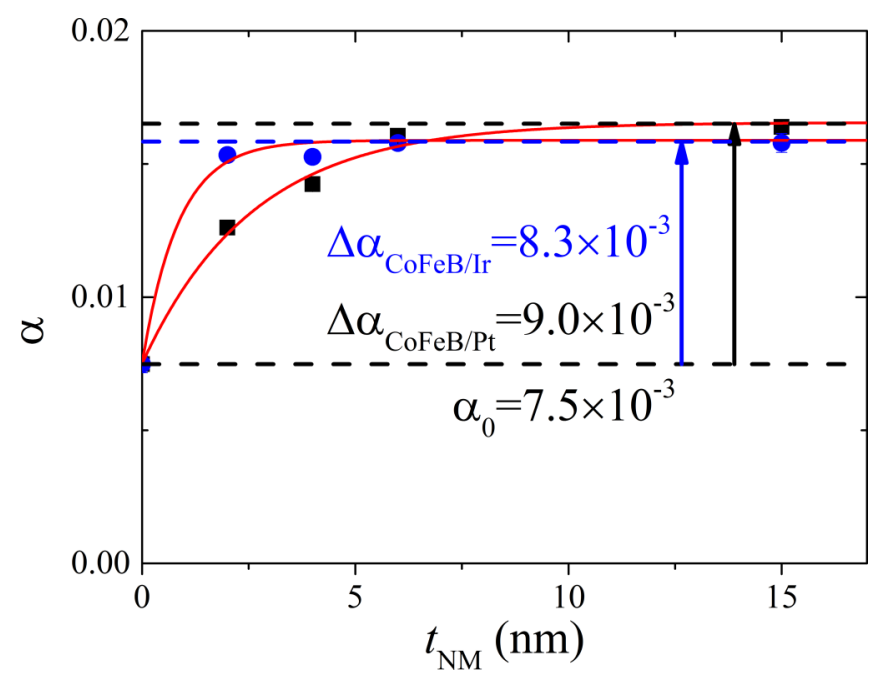

FIG. 3. Evolution of the deduced magnetic damping $\alpha$ from SP-FMR as a function of the thickness of the nonmagnetic material (Ir or Pt) on $/ / \operatorname{CoFeB}(5 \mathrm{~nm}) / \mathrm{Ir}$ or $\mathrm{Pt}\left(t_{N M} \mathrm{~nm}\right)$. In the case where $t_{N M}=0$, the magnetic damping value is obtained from FMR measurements. Red curves stand for an exponential decay, $\alpha=$ $\Delta \alpha\left(1-e^{-t_{N M} / l_{d}}\right)$. It results that $l_{d}=2.6 \pm 0.3(0.9 \pm 0.2) \mathrm{nm}$ for $\mathrm{CoFeB} / \mathrm{Pt}(\mathrm{CoFeB} / \mathrm{Ir})$. Note that there is not a factor 2 in the exponential argument.

magnetic layer probed by FMR in order to obtain the intrinsic damping.

As we can see in Fig. 3, the magnetic damping increases strongly for both capping layers, Pt as well as Ir. This phenomenon is a well-known feature of damping enhancement due to the spin pumping effect $[19,26,29]$, and can be characterized by the following relation:

$$
\Delta \alpha=\alpha-\alpha_{0}=\frac{g \mu_{B} g_{\text {eff }}^{\uparrow \downarrow}}{4 \pi M_{S} t_{F M}},
$$

where $g$ is the Landé factor ( 2.11 for $\mathrm{CoFeB}), g_{\text {eff }}^{\uparrow \downarrow}$ is the real part of the effective spin mixing conductance, $\mu_{B}$ is the Bohr magneton, and $t_{F M}$ is the ferromagnetic ( $\mathrm{CoFeB}$ in our case) layer thickness. Replacing our experimental values in Eq. (4), we estimated the value of $g_{\text {eff }}^{\uparrow \downarrow}$ for the $\mathrm{Ir} / \mathrm{CoFeB}$ interface to be around $30 \mathrm{~nm}^{-2}$, and $32 \mathrm{~nm}^{-2}$ for the $\mathrm{Pt} / \mathrm{CoFeB}$ interface. These values are in the typical order of magnitude of effective spin mixing conductances obtained for ferromagnetic/Pt systems (for Py/Pt: $21-30 \mathrm{~nm}^{-2}$ [23,29,31,33]; for Co/Pt: $80 \mathrm{~nm}^{-2}$ [26]) and epitaxial Fe/Pt: $26 \mathrm{~nm}^{-2}$ [43]. Especially, for the $\mathrm{CoFeB} / \mathrm{Pt}$ interface, some reported values are $40 \mathrm{~nm}^{-2}$ [44], $54 \mathrm{~nm}^{-2}$, and $47 \mathrm{~nm}^{-2}$ for the opposite stacking order, $\mathrm{Pt} / \mathrm{CoFeB}$, in [45] and $50.7 \mathrm{~nm}^{-2}$ in [46]). In the case of Ir has been reported for NiFe/Ir interfaces so far $13 \mathrm{~nm}^{-2}$ in [17] and $25.2 \mathrm{~nm}^{-2}$ in [18]. Those values are effective values since they include interface contributions such a spin memory loss $[18,26]$. Let us point out here that we do not consider the imaginary part of the spin mixing conductance in our work, since the Kittel fittings performed do not show a value of the gyromagnetic ratio differing from the one of electrons, as predicted for metallic systems $[19,47]$.

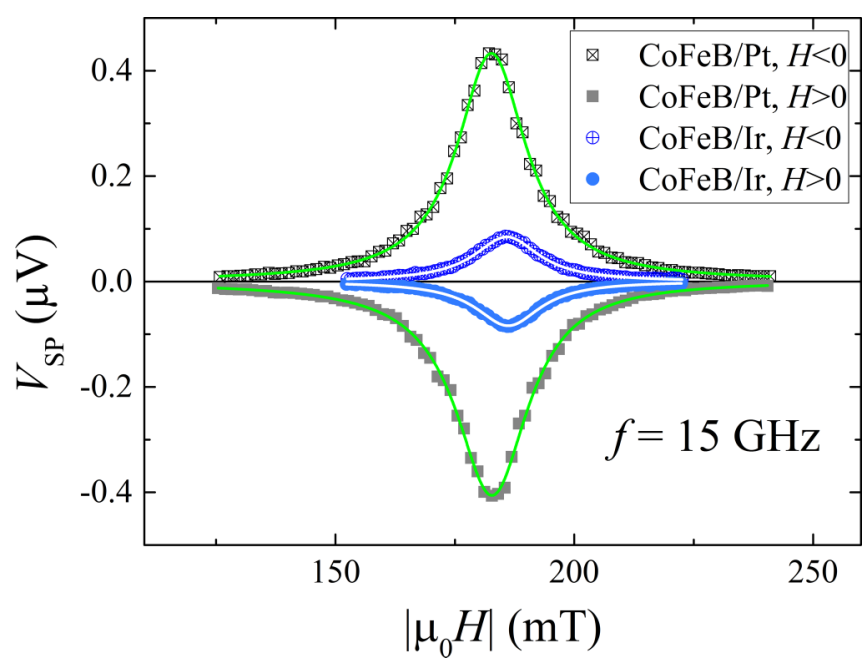

FIG. 4. Spin pumping voltage $V_{\text {sp }}$ for $\mathrm{Si} / \mathrm{SiO}_{2} / \mathrm{CoFeB}(5 \mathrm{~nm}) / \mathrm{Ir}$ $(6 \mathrm{~nm})$ and $\mathrm{Si} / \mathrm{SiO}_{2} / \mathrm{CoFeB}(5 \mathrm{~nm}) / \mathrm{Pt}(6 \mathrm{~nm})$ as a function of the applied field absolute value $(|H|)$ for an excitation frequency of $15 \mathrm{GHz}$. Results are shown for positive and negative static applied fields. Symbols represent experimental measurements whereas the full lines correspond to a fit of the data by a Lorentzian function. A constant offset was subtracted. The raw voltage is purely symmetrical, getting rid of the spurious signals.

\section{SPIN DIFFUSION LENGTH AND SPIN HALL ANGLE DETERMINATION}

Finally, the spin pumping voltage measured normalized by the resistance of the FM/NM slab gives the charge current produced by ISHE. In this geometry the charge current measured can be expressed as follows [26,27,29]:

$$
I_{C}=\theta_{S H E} l_{s f} w J_{S}^{\mathrm{eff}} \tanh \left(\frac{t_{N M}}{2 l_{s f}}\right)
$$

where $w=10 \mu \mathrm{m}$ is the width of the device. Here, $J_{S}^{\text {eff }}$ is the effective spin current density injected in the NM layer and it follows the relationship [26,27]

$$
J_{S}^{\mathrm{eff}}=\frac{e g_{\mathrm{eff}}^{\uparrow \downarrow} \gamma^{2} h_{R F}^{2}}{4 \pi \alpha^{2}} A(\omega)
$$

where $A(\omega)=\frac{\gamma \mu_{0} M_{\text {eff }}+\sqrt{\left(\gamma \mu_{0} M_{\text {eff }}\right)^{2}+4 \omega^{2}}}{\left(\gamma \mu_{0} M_{\text {eff }}\right)^{2}+4 \omega^{2}}$ represents the influence of the magnetic dynamics on the injected spin currents, as it was shown by Ando et al. [27]. Figure 4 shows the raw data of spin pumping voltage $\left(V_{\mathrm{sp}}\right)$ as a function of the applied magnetic field $\left(\mu_{0} H\right)$ for an excitation frequency of $15 \mathrm{GHz}$. We can observe that the voltage is a purely symmetric Lorenztian around the resonance field and it changes its sign upon changing the sign of the applied field. All these are features of an ISHE spin pumping voltage. Furthemore, that is also verified in the case of $\mathrm{CoFeB} / \mathrm{Pt}$ bilayer.

Figure 5 shows the evolution of the charge current produced as a function of the iridium thickness in $\mathrm{CoFeB} / \mathrm{Ir}$ bilayers for various frequencies. Using these results, the spin diffusion length for Iridium, $l_{s f}^{I r}$, can be deduced from Eq. (5). Thus, $l_{s f}^{I r}$ obtained for each frequency is displayed in Fig. 6 . 


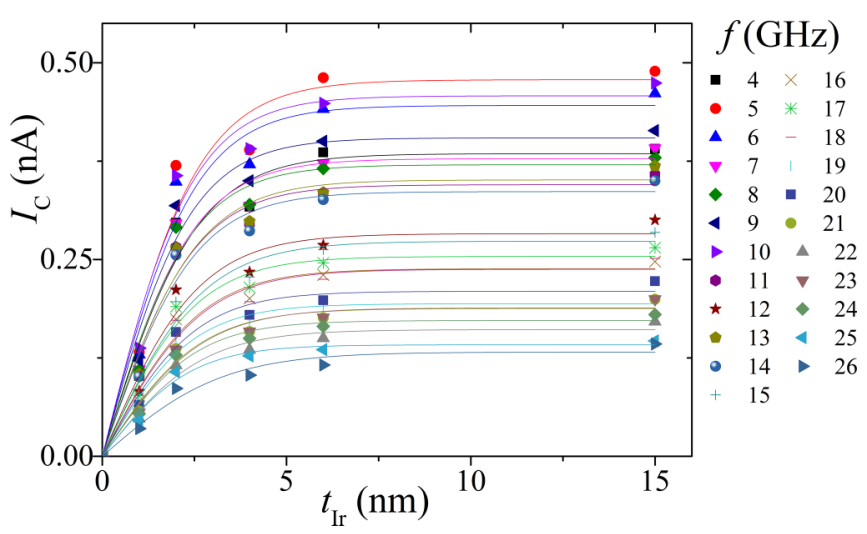

FIG. 5. Produced charge current $\left(I_{\mathrm{c}}\right)$ as a function of the iridium thickness $\left(t_{\text {Ir }}\right)$ for frequencies ranging from 4 to $26 \mathrm{GHz}$. The symbols represent experimental values whereas the solid lines show the fitting obtained thanks to Eq. (5).

Our results show consistent values of $l_{s f}^{I r}=1.3 \pm 0.1 \mathrm{~nm}$ and $l_{s f}^{P t}=2.4 \pm 0.3 \mathrm{~nm}$ (red dashed lines).

The value of spin diffusion length obtained for platinum is in agreement with the values found in the literature: the experimental values reported using the SP-FMR setup range from $0.5 \mathrm{~nm}$ [32] to $10 \mathrm{~nm}$ [23], with numerous values in between $[10,48]$. The obtained value $l_{s f}^{I r}=1.3 \mathrm{~nm}$ is twice larger than the one presented in earlier studies $[17,18]$ with similar FMR-based methods, and close to the one reported by spin-orbit torque technique, $\sim 1 \mathrm{~nm}$ [7]. That difference might be due to different Ir resistivity. However, we would like to note that $l_{\mathrm{sf}}$ values reported only by spin pumping FMR measurements (not spin pumping voltage measurements) consider an exponential decay of damping with and argument $\left(2 l_{\mathrm{sf}} / t_{\mathrm{NM}}\right)$. However, the $t_{\mathrm{NM}}$ damping evolution is not reliable to estimate $l_{\text {sf }}$ as it was pointed out in Ref. [26]. We can observe that discrepancy with results in Fig. 3 where $l_{\mathrm{d}}$ is close to $l_{\text {sf }}$ estimated by charge current dependence in Figs. 5 and 6 but we have used a different exponential argument $\left(l_{\mathrm{d}} / t_{\mathrm{NM}}\right)$.

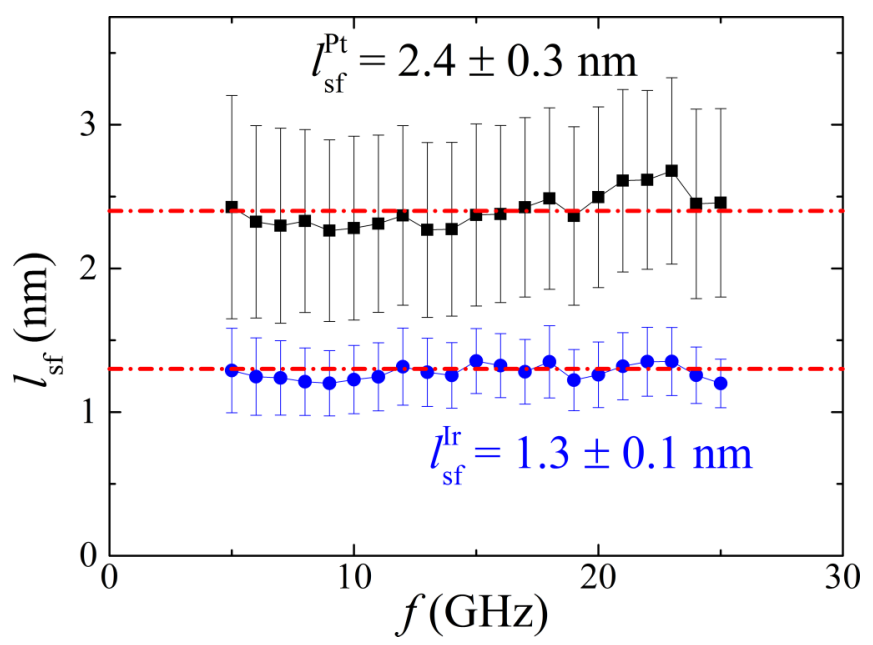

FIG. 6. Spin diffusion length $l_{s f}$ as a function of the frequency in iridium and platinum deduced from the fit shown in Fig. 5.
This is likely to explain the difference with the two previous studies $[17,18]$.

Further, $l_{s f}^{I r}=1.3 \mathrm{~nm}$ can be compared to the usual range of thicknesses where iridium is used, especially in the case of synthetic ferrimagnets where the iridium spacer is used to maximize the RKKY coupling, around 0.5 or $1.5 \mathrm{~nm}$ (first and second peaks) $[2,8,9]$. From the experimental values of spin diffusion length and resistivity of the HM layer, we can compute its spin resistance $r_{s}=\rho \times l_{s f}$. The resistivities measured for Pt and Ir are the following: $\rho_{P t}=245 \mathrm{n} \Omega \mathrm{m}$ and $\rho_{I r}=250 \mathrm{n} \Omega \mathrm{m}$. We thus have the spin resistance $r_{s, P t}=$ $0.59 \mathrm{f} \Omega \mathrm{m}^{2}$ and $r_{s, I r}=0.32 \mathrm{f} \Omega \mathrm{m}^{2}$. The value of $r_{s, P t}$ is very close to the experimental result published in Ref. [26] as well as close to the theoretical value reported by Liu et al. [49]. We can also use the remark from Ref. [26], stating that in the case of Pt, given the results reported in the literature, the product of the effective spin Hall angle and the spin diffusion length, $\theta_{S H} \times l_{s f}$, is a quantity that is nearly independent of the technique or the setup used, and its effective value is estimated to be close to $0.19 \mathrm{~nm}$. It is therefore possible to obtain the effective spin Hall angle of platinum, leading to a value of $\theta_{S H}^{P t} \approx 7.6 \%$. Moreover, our results of shorter spin diffusion length on the more resistive material, Ir, also confirm that the predominant spin-relaxation contribution is due to the Elliot-Yafet mechanism. Indeed with an increase in resistivity, the spin-relaxation rate via the Elliot-Yafet mechanism also increases [50,51].

To determine $\theta_{S H}^{I r}$ accurately and independently, the value of the effective spin current is needed. In order to do so, it is mandatory to estimate the strength of the radio-frequency excitation field $h_{\mathrm{rf}}$ and its frequency dependence, as well as the $g_{\text {eff }}^{\uparrow \downarrow}$ factor for the $\mathrm{CoFeB} / \mathrm{Ir}$ interface. The latter was previously estimated to $30 \mathrm{~nm}^{2}$. After accurate measurements of the transmission line and of the scattering matrices of the devices corresponding to our samples, we have concluded that the frequency dependence of $h_{\mathrm{RF}}$ with respect to the frequency of the signal is the same for the iridium and the platinum based samples. This was expected, since the values of the conductivities are found to be very close for both materials.

Now, we defined the quantity

$$
\Im_{S P}=\frac{I_{C} \alpha^{2}}{l_{s f} g_{\text {eff }}^{\uparrow \downarrow} A(\omega)} .
$$

We can then plot the ratio $\frac{\Im_{S P}^{I r}}{\Im_{S P P}^{P}}$ of this $\Im$ parameter given in Eq. (7) for different frequencies experimentally measured as displayed in Fig. 7. This $\frac{\Im_{S P}^{I r}}{\Im_{S P}^{P T}}$ value is most likely to give the right estimate of the actual spin Hall angle ratios, since it can be interpreted as

$$
\frac{\Im_{S P}^{I r}}{\Im_{S P}^{P t}}=\frac{\theta_{S H}^{I r}}{\theta_{S H}^{P t}} \frac{\tanh \left[t_{I r} /\left(2 l_{s f}^{I r}\right)\right]}{\tanh \left[t_{P t} /\left(2 l_{s f}^{P t}\right)\right]} .
$$

The limit obtained for $t_{N M} \gg l_{s f}^{N M}$ is the ratio of spin Hall angles. Indeed, we can represent this ratio as a function of the nonmagnetic materials thickness as shown in Fig. 8. We can observe a very large discrepancy between the value of the ratio given in Eq. (8) and the one obtained experimentally for $t_{\mathrm{NM}}=2 \mathrm{~nm}$. 


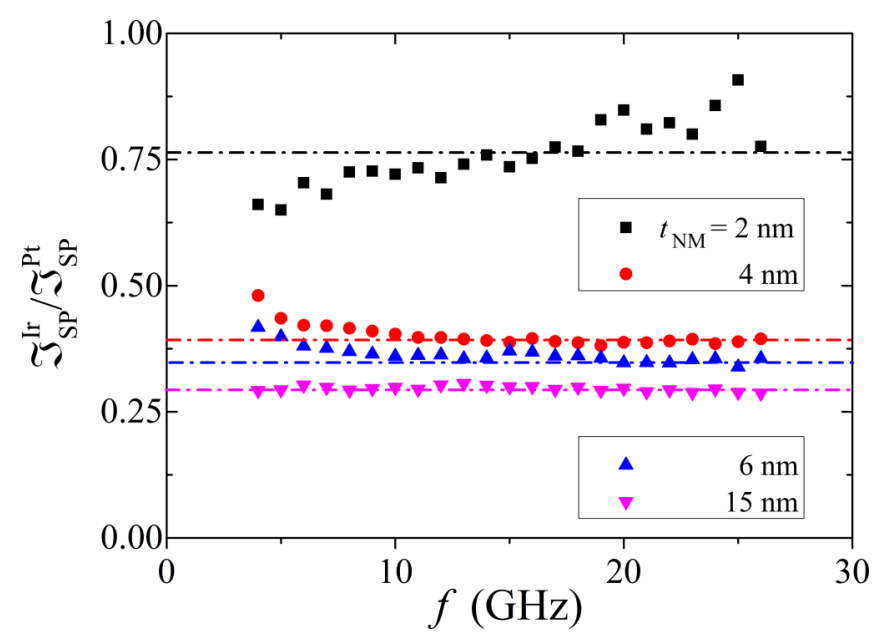

FIG. 7. Ratio $\Im$ of the spin pumping currents based on Eq. (7) as a function of the frequency. The dashed lines are guides towards the value obtained at high frequency.

Numerous elements can explain the difference between the model given in Eq. (8) and the experimental results at low thickness. First, we can question the validity of the assumptions used in our study. We have considered that the resistivity, the spin diffusion length, and the spin Hall angle of the materials were independent of the nonmagnetic material thickness. However, this approximation does not hold for very low thicknesses, which is where the model and the experimental results do not match. Furthermore, at very low thicknesses, the roughness and the quality of the interface plays a larger role than for thick layers. The errors on the thicknesses and on the ratios are expected to be larger than for thicker samples.

Nevertheless, a good agreement for nonmagnetic materials thickness superior to $2 \mathrm{~nm}$ is obtained, and we can estimate the ratios of effective SHE efficiencies to be $\frac{\theta_{S H}^{l r}}{\theta_{S H}^{P H}}=0.26$. This approach lets us evaluate the values of $l_{\mathrm{sf}}$ and $\theta_{\mathrm{SH}}$ with precision.

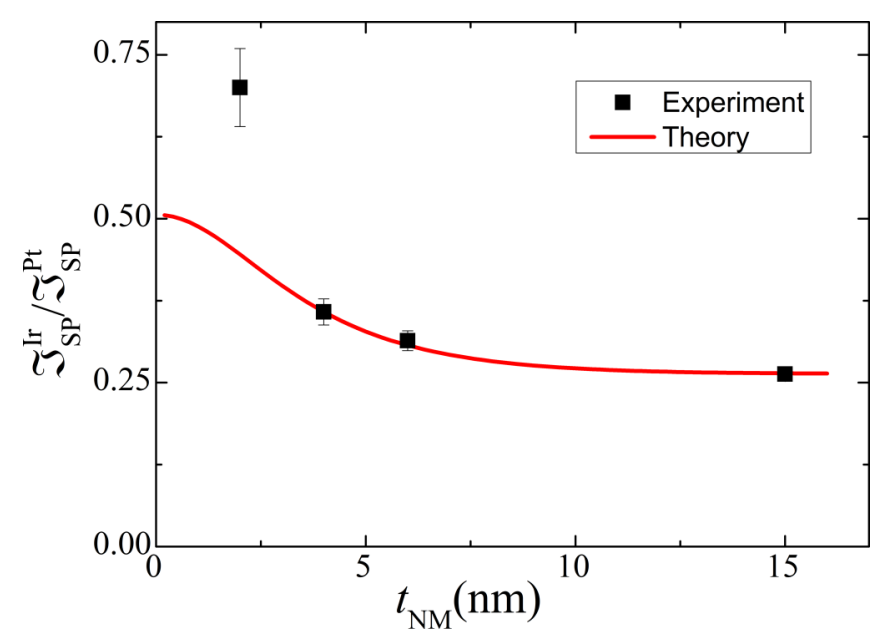

FIG. 8. Evolution of the corrected spin pumping currents ratio as a function of the nonmagnetic materials thickness (black squares) and the expected dependence (red line) according to Eq. (8).
Besides, using our determination of the spin Hall angle of platinum at $7.6 \%$, we can estimate the spin Hall angle of iridium to be around $2 \%$. Literature provides a large range of values for Pt that span to more than an order of magnitude, ranging from 0.33 to 0.0067 [22,23,32-35,47,48,24-31]. Our result for Ir is in good agreement with what was found in Ref. [17], with a $2 \%$ value, and twice the one reported by spin-orbit torque in Ref. [7]. The method that we present here enables us to make a comparison by getting rid of many artefacts that seem to be the cause of a broadening of the results obtained in the literature.

Furthermore, our results clearly show that the spin Hall angle sign of $\mathrm{Pt}$ and Ir are the same. This confirms the simple picture of the SOC sign in transition metals. Thus $\lambda_{\text {SOC }}<0$ for a $d$ band less than half full and $\lambda_{\text {SOC }}>0$ for a $d$ band more than half full. The last case corresponds to $\operatorname{Ir}\left(5 d^{9} 6 s^{0}\right)$ and $\operatorname{Pt}\left(5 d^{9} 6 s^{1}\right)$ in solid states and agrees with theoretical calculation of positive spin Hall conductivities, $\sigma_{\mathrm{SHC}}$ [52]. Moreover, from our data, $\sigma_{\mathrm{SHC}}=\theta_{\mathrm{SHE}} / \rho$ results 3100 (800) $\Omega^{-1} \mathrm{~cm}^{-1}$ for Pt (Ir). We obtain a slightly lower result than that calculated for Pt in Ref. [26], $3240 \Omega^{-1} \mathrm{~cm}^{-1}$, performing also ISHE-SP-FMR experiments.

We can use the works in Ref. [53] to evaluate the efficiency of these two materials for spin-to-charge and charge-to-spin conversion applications. For the generation of a charge current, the figure of merit proposed is the product $\lambda_{I S H E}^{*}=$ $\theta_{S H} \times l_{s f}$. We find a value of $0.186 \mathrm{~nm}$ for $\mathrm{Pt}$, and 0.026 $\mathrm{nm}$ for Ir, suggesting that iridium is a poor candidate for further spin pumping applications. However, if we consider the figure of merit to assess the spin current generation, which is mandatory for spin-orbit torque (SOT), given by the formula $q^{*}=0.38 \times \frac{\theta_{S H}}{l_{s f}}$, we find a value of $12 \times 10^{-3} \mathrm{~nm}^{-1}$ for $\mathrm{Pt}$ and $\sim 6 \times 10^{-3} \mathrm{~nm}^{-1}$ for Ir. Therefore, it appears that even though $\mathrm{Pt}$ is the best material among those studied in both cases, Ir is good candidate, with half the ability of Pt to generate efficiently a spin current.

\section{CONCLUSION}

In this paper, we have described an approach that enables the measurement of the spin Hall angle of a material with respect to another one. We report reliable values of spin diffusion lengths of $1.3 \pm 0.1 \mathrm{~nm}$ for iridium and $2.4 \pm 0.3 \mathrm{~nm}$ for platinum from the NM thickness dependence of the charge current (and not from damping evolution). The spin mixing conductances for both interfaces $\mathrm{CoFeB} / \mathrm{Ir}$ and $\mathrm{CoFeB} / \mathrm{Pt}$ have been estimated around 30 and $32 \mathrm{~nm}^{-2}$, respectively. The spin Hall angle of Ir has the same sign as the one of Pt and represents $26 \%$ of its value. We could obtain a $\theta_{\mathrm{SH}}$ value of $7.6 \%$ for Pt, from which we could deduce a $\theta_{\mathrm{SH}}$ value of $2 \%$ for Ir. Even though this procedure does not give by itself the value of the spin Hall characteristics of a material, it gives information about materials in the same conditions, and enables a comparison between various materials. This can be an opportunity to unify the results concerning spin diffusion lengths and spin Hall angles, given the large dispersion in the results reported in the past decade. The spintronic parameters we are reporting for Ir will appeal for more applications exploiting this material in new spin-orbitronic devices such 
as combined spin-orbit torque and spin transfer torque effects in magnetic tunnel junctions [54]. This is by combining two major effects in spintronics, RKKY and SHE.

\section{ACKNOWLEDGMENTS}

T.F. thanks the ANRT and the company Vinci Technologies for funding his Ph.D., under CIFRE Convention No. 2016/1458. All authors acknowledge support from Agence Nationale de la Recherche (France) under Contracts
No. ANR-18-CE24-0008 (MISSION) and No. ANR-19CE24-0016-01 (TOPTRONIC) from the French PIA project "Lorraine Université d'Excellence," Reference No. ANR15IDEX-04-LUE, from Region Grand Est, Metropole du Grand Nancy, Institut Carnot ICEEL, from the "FEDER-FSE Lorraine et Massif Vosges 2014-2020," a European Union Program. Devices in the present study were patterned at MiNaLor clean-room platform which is partially supported by FEDER and Grand Est Region through the RaNGE project.
[1] J. P. Clancy, N. Chen, C. Y. Kim, W. F. Chen, K. W. Plumb, B. C. Jeon, T. W. Noh, and Y. J. Kim, Spin-orbit coupling in iridium-based $5 \mathrm{~d}$ compounds probed by $\mathrm{x}$-ray absorption spectroscopy, Phys. Rev. B 86, 195131 (2012).

[2] S. S. P. Parkin, Systematic Variation of the Strength and Oscillation Period of Indirect Magnetic Exchange Coupling Through the 3d, 4d, and 5d Transition Metals, Phys. Rev. Lett. 67, 3598 (1991).

[3] K. Nakamura, T. Nomura, A.-M. Pradipto, K. Nawa, T. Akiyama, and T. Ito, Effect of heavy-metal insertions at $\mathrm{Fe} / \mathrm{MgO}$ interfaces on electric-field-induced modification of magnetocrystalline anisotropy, J. Magn. Magn. Mater. 429, 214 (2017).

[4] P. Taivansaikhan, D. Odkhuu, S. H. Rhim, and S. C. Hong, Gigantic perpendicular magnetic anisotropy of heavy transition metal cappings on $\mathrm{Fe} / \mathrm{MgO}(000$ 1), J. Magn. Magn. Mater. 442, 183 (2017).

[5] T. Nozaki, A. Kozioł-Rachwał, M. Tsujikawa, Y. Shiota, X. Xu, T. Ohkubo, T. Tsukahara, S. Miwa, M. Suzuki, S. Tamaru, H. Kubota, A. Fukushima, K. Hono, M. Shirai, Y. Suzuki, and S. Yuasa, Highly efficient voltage control of spin and enhanced interfacial perpendicular magnetic anisotropy in iridium-doped $\mathrm{Fe} / \mathrm{MgO}$ magnetic tunnel junctions, NPG Asia Mater. 9, e451 (2017).

[6] Y.-C. Lau, Z. Chi, T. Taniguchi, M. Kawaguchi, G. Shibata, N. Kawamura, M. Suzuki, S. Fukami, A. Fujimori, H. Ohno, and M. Hayashi, Giant perpendicular magnetic anisotropy in Ir/Co/Pt multilayers, Phys. Rev. Mater. 3, 104419 (2019).

[7] Y. Ishikuro, M. Kawaguchi, N. Kato, Y.-C. Lau, and M. Hayashi, Dzyaloshinskii-Moriya interaction and spin-orbit torque at the Ir/Co interface, Phys. Rev. B 99, 134421 (2019).

[8] T. Fache, H. S. Tarazona, J. Liu, G. L'vova, M. J. Applegate, J. C. Rojas-Sanchez, S. Petit-Watelot, C. V. Landauro, J. Quispe-Marcatoma, R. Morgunov, C. H. W. Barnes, and S. Mangin, Nonmonotonic aftereffect measurements in perpendicular synthetic ferrimagnets, Phys. Rev. B 98, 064410 (2018).

[9] B. Böhm, L. Fallarino, D. Pohl, B. Rellinghaus, K. Nielsch, N. S. Kiselev, and O. Hellwig, Antiferromagnetic domain wall control via surface spin flop in fully tunable synthetic antiferromagnets with perpendicular magnetic anisotropy, Phys. Rev. B 100, 140411(R) (2019).

[10] A. Hoffmann, Spin hall effects in metals, IEEE Trans. Magn. 49, 5172 (2013).

[11] L. Liu, C.-F. Pai, Y. Li, H. W. Tseng, D. C. Ralph, and R. A. Buhrman, Spin-torque switching with the giant spin hall effect of tantalum, Science 336, 555 (2012).
[12] A. Brataas, A. D. Kent, and H. Ohno, Current-induced torques in magnetic materials, Nat. Mater. 11, 372 (2012).

[13] T. Jungwirth, J. Wunderlich, and K. Olejník, Spin Hall effect devices, Nat. Mater. 11, 382 (2012).

[14] I. Mihai Miron, K. Garello, G. Gaudin, P.-J. Zermatten, M. V. Costache, S. Auffret, S. Bandiera, B. Rodmacq, A. Schuhl, and P. Gambardella, Perpendicular switching of a single ferromagnetic layer induced by in-plane current injection, Nature (London) 476, 189 (2011).

[15] T. Kimura, Y. Otani, T. Sato, S. Takahashi, and S. Maekawa, Room-Temperature Reversible Spin Hall Effect, Phys. Rev. Lett. 98, 156601 (2007).

[16] J. E. Hirsch, Spin Hall Effect, Phys. Rev. Lett. 83, 1834 (1999).

[17] W. Zhang, M. B. Jungfleisch, W. Jiang, J. Sklenar, F. Y. Fradin, J. E. Pearson, J. B. Ketterson, and A. Hoffmann, Spin pumping and inverse spin Hall effects-Insights for future spinorbitronics (invited), J. Appl. Phys. 117, 172610 (2015).

[18] T. White, T. Bailey, M. Pierce, and C. W. Miller, Strong spin pumping in permalloy-iridium heterostructures, IEEE Magn. Lett. 8, 1 (2017).

[19] Y. Tserkovnyak, A. Brataas, G. E. W. Bauer, and B. I. Halperin, Nonlocal magnetization dynamics in ferromagnetic heterostructures, Rev. Mod. Phys. 77, 1375 (2005).

[20] H. J. Jiao and G. E. W. Bauer, Spin Backflow and ac Voltage Generation by Spin Pumping and the Inverse Spin Hall Effect, Phys. Rev. Lett. 110, 217602 (2013).

[21] L. Bai, P. Hyde, Y. S. Gui, C.-M. Hu, V. Vlaminck, J. E. Pearson, S. D. Bader, and A. Hoffmann, Universal Method for Separating Spin Pumping from Spin Rectification Voltage of Ferromagnetic Resonance, Phys. Rev. Lett. 111, 217602 (2013).

[22] E. Saitoh, M. Ueda, H. Miyajima, and G. Tatara, Conversion of spin current into charge current at room temperature: Inverse spin-Hall effect, Appl. Phys. Lett. 88, 182509 (2006).

[23] O. Mosendz, V. Vlaminck, J. E. Pearson, F. Y. Fradin, G. E. W. Bauer, S. D. Bader, and A. Hoffmann, Detection and quantification of inverse spin Hall effect from spin pumping in permalloy/normal metal bilayers, Phys. Rev. B 82, 214403 (2010).

[24] C. Hahn, G. de Loubens, O. Klein, M. Viret, V. V. Naletov, and J. Ben Youssef, Comparative measurements of inverse spin Hall effects and magnetoresistance in YIG/Pt and YIG/Ta, Phys. Rev. B 87, 174417 (2013).

[25] K. Kondou, H. Sukegawa, S. Mitani, K. Tsukagoshi, and S. Kasai, Evaluation of spin hall angle and spin diffusion length by using spin current-induced ferromagnetic resonance, Appl. Phys. Express 5, 073002 (2012).

[26] J.-C. Rojas-Sánchez, N. Reyren, P. Laczkowski, W. Savero, J.-P. Attané, C. Deranlot, M. Jamet, J.-M. George, L. Vila, and H. 
Jaffrès, Spin Pumping and Inverse Spin Hall Effect in Platinum: The Essential Role of Spin-Memory Loss at Metallic Interfaces, Phys. Rev. Lett. 112, 106602 (2014).

[27] K. Ando, S. Takahashi, J. Ieda, Y. Kajiwara, H. Nakayama, T. Yoshino, K. Harii, Y. Fujikawa, M. Matsuo, S. Maekawa, and E. Saitoh, Inverse spin-Hall effect induced by spin pumping in metallic system, J. Appl. Phys. 109, 103913 (2011).

[28] L. Liu, T. Moriyama, D. C. Ralph, and R. A. Buhrman, SpinTorque Ferromagnetic Resonance Induced by the Spin Hall Effect, Phys. Rev. Lett. 106, 036601 (2011).

[29] A. Azevedo, L. H. Vilela-Leão, R. L. Rodríguez-Suárez, A. F. Lacerda Santos, and S. M. Rezende, Spin pumping and anisotropic magnetoresistance voltages in magnetic bilayers: Theory and experiment, Phys. Rev. B 83, 144402 (2011).

[30] Z. Feng, J. Hu, L. Sun, B. You, D. Wu, J. Du, W. Zhang, A. Hu, Y. Yang, D. M. Tang, B. S. Zhang, and H. F. Ding, Spin Hall angle quantification from spin pumping and microwave photoresistance, Phys. Rev. B 85, 214423 (2012).

[31] H. Nakayama, K. Ando, K. Harii, T. Yoshino, R. Takahashi, Y. Kajiwara, K. Uchida, Y. Fujikawa, and E. Saitoh, Geometry dependence on inverse spin Hall effect induced by spin pumping in $\mathrm{Ni}_{81} \mathrm{Fe}_{19} / \mathrm{Pt}$ films, Phys. Rev. B 85, 144408 (2012).

[32] C. T. Boone, H. T. Nembach, J. M. Shaw, and T. J. Silva, Spin transport parameters in metallic multilayers determined by ferromagnetic resonance measurements of spin-pumping, J. Appl. Phys. 113, 153906 (2013).

[33] M. Obstbaum, M. Härtinger, H. G. Bauer, T. Meier, F. Swientek, C. H. Back, and G. Woltersdorf, Inverse spin Hall effect in $\mathrm{Ni}_{81} \mathrm{Fe}_{19}$ normal-metal bilayers, Phys. Rev. B 89, 060407(R) (2014).

[34] V. Castel, N. Vlietstra, J. Ben Youssef, and B. J. van Wees, Platinum thickness dependence of the inverse spinHall voltage from spin pumping in a hybrid yttrium iron garnet/platinum system, Appl. Phys. Lett. 101, 132414 (2012).

[35] O. D’Allivy Kelly, A. Anane, R. Bernard, J. Ben Youssef, C. Hahn, A. H. Molpeceres, C. Carrétéro, E. Jacquet, C. Deranlot, P. Bortolotti, R. Lebourgeois, J. C. Mage, G. De Loubens, O. Klein, V. Cros, and A. Fert, Inverse spin Hall effect in nanometer-thick yttrium iron garnet/Pt system, Appl. Phys. Lett. 103, 082408 (2013).

[36] C. Kittel, Ferromagnetic resonance, J. Phys. Rad. 12, 291 (1951).

[37] J.-M. Beaujour, D. Ravelosona, I. Tudosa, E. E. Fullerton, and A. D. Kent, Ferromagnetic resonance linewidth in ultrathin films with perpendicular magnetic anisotropy, Phys. Rev. B 80, 180415(R) (2009).

[38] C. Bilzer, T. Devolder, J.-V. Kim, G. Counil, C. Chappert, S. Cardoso, and P. P. Freitas, Study of the dynamic magnetic properties of soft CoFeB films, J. Appl. Phys. 100, 53903 (2006).

[39] X. Liu, W. Zhang, M. J. Carter, and G. Xiao, Ferromagnetic resonance and damping properties of $\mathrm{CoFeB}$ thin films as free layers in $\mathrm{MgO}$-based magnetic tunnel junctions, J. Appl. Phys. 110, 33910 (2011).
[40] A. Conca, J. Greser, T. Sebastian, S. Klingler, B. Obry, B. Leven, and B. Hillebrands, Low spin-wave damping in amorphous $\mathrm{Co}_{40} \mathrm{Fe}_{40} \mathrm{~B}_{20}$ thin films, J. Appl. Phys. 113, 213909 (2013).

[41] A. Conca, E. T. Papaioannou, S. Klingler, J. Greser, T. Sebastian, B. Leven, J. Lösch, and B. Hillebrands, Annealing influence on the Gilbert damping parameter and the exchange constant of CoFeB thin films, Appl. Phys. Lett. 104, 182407 (2014).

[42] F. Xu, Q. Huang, Z. Liao, S. Li, and C. K. Ong, Tuning of magnetization dynamics in sputtered $\mathrm{CoFeB}$ thin film by gas pressure, J. Appl. Phys. 111, 07A304 (2012).

[43] C. Guillemard, S. Petit-Watelot, S. Andrieu, and J.-C. RojasSánchez, Charge-spin current conversion in high quality epitaxial Fe/Pt systems: Isotropic spin Hall angle along different in-plane crystalline directions, Appl. Phys. Lett. 113, 262404 (2018).

[44] A. Ruiz-Calaforra, T. Brächer, V. Lauer, P. Pirro, B. Heinz, M. Geilen, A. V. Chumak, A. Conca, B. Leven, and B. Hillebrands, The role of the non-magnetic material in spin pumping and magnetization dynamics in $\mathrm{NiFe}$ and $\mathrm{CoFeB}$ multilayer systems, J. Appl. Phys. 117, 163901 (2015).

[45] C. Swindells, A. T. Hindmarch, A. J. Gallant, and D. Atkinson, Spin transport across the interface in ferromagnetic/nonmagnetic systems, Phys. Rev. B 99, 064406 (2019).

[46] D. J. Kim, S. Il Kim, S. Y. Park, K. D. Lee, and B. G. Park, Ferromagnetic resonance spin pumping in $\mathrm{CoFeB}$ with highly resistive non-magnetic electrodes, Curr. Appl. Phys. 14, 1344 (2014).

[47] W. Skowroński, Ł. Karwacki, S. Ziętek, J. Kanak, S. Łazarski, K. Grochot, T. Stobiecki, P. Kuświk, F. Stobiecki, and J. Barnaś, Determination of Spin Hall Angle in Heavy-Metal/Co-Fe-BBased Heterostructures with Interfacial Spin-Orbit Fields, Phys. Rev. Appl. 11, 024039 (2019).

[48] J. Sinova, S. O. Valenzuela, J. Wunderlich, C. H. Back, and T. Jungwirth, Spin Hall effects, Rev. Mod. Phys. 87, 1213 (2015).

[49] Y. Liu, Z. Yuan, R. J. H. Wesselink, A. A. Starikov, and P. J. Kelly, Interface Enhancement of Gilbert Damping from First Principles, Phys. Rev. Lett. 113, 207202 (2014).

[50] R. J. Elliott, Theory of the effect of spin-Orbit coupling on magnetic resonance in some semiconductors, Phys. Rev. 96, 266 (1954).

[51] Y. Yafet, g Factors and spin-lattice relaxation of conduction electrons, Solid State Phys. 14, 1 (1963).

[52] T. Tanaka, H. Kontani, M. Naito, T. Naito, D. S. Hirashima, K. Yamada, and J. Inoue, Intrinsic spin Hall effect and orbital Hall effect in 4d and 5d transition metals, Phys. Rev. B 77, 165117 (2008).

[53] J. C. Rojas-Sánchez and A. Fert, Compared Efficiencies of Conversions between Charge and Spin Current by Spin-Orbit Interactions in Two- and Three-Dimensional Systems, Phys. Rev. Appl. 11, 054049 (2019).

[54] E. Grimaldi, V. Krizakova, G. Sala, F. Yasin, S. Couet, G. Sankar Kar, K. Garello, and P. Gambardella, Single-shot dynamics of spin-orbit torque and spin transfer torque switching in three-terminal magnetic tunnel junctions, Nat. Nanotechnol. 15, 111 (2020). 